2. Zimmerman R, Spann S. Poliovirus vaccine options. Am Fam Physician. 1999;59(1):113-8, 125-6.

3. Andrus J, Strebel P, de Quadros C, Olivé J. Risk of vaccine-associated paralytic poliomyelitis in Latin America, 1989-91. Bull World Health Organ. 1995;73(1):33-40.

4. Organización Mundial de la Salud. Polio vaccines and routine polio immunization in the pre-eradication era. position paper, Draft 2 Mar 2010, external review. Meeting of the Strategic Advisory Group of Experts on Immunization (SAGE). Geneva, WHO. April 2010.

5. Ministerio de Salud del Perú, Dirección General de Epidemiología. Informe sobre situación de la poliomielitis en el Perú, 2011. Lima: Minsa; 2011.

6. Organización Panamericana de la Salud. Alerta Epidemiológica, 20 de octubre del 2010. Washington, DC: OPS; 2010.

7. Organización Panamericana de la Salud. Programa Ampliado de Inmunizaciones. Precio de las vacunas para el 2011. Enmienda III, 27-6-2011. Washington, DC: OPS; 2011.

Correspondencia: Wilfredo Gutiérrez Peñafiel

Dirección: Av Lima 270, Block 31 Dpto 503, Barranco

Teléfono: (51) 989723100

Correo electrónico: gutierrw@gmail.com

\section{SUBREGISTRO DE TRASTORNOS DEPRESIVOS}

\section{UNDER REPORTING OF DEPRESSIVE DISORDERS}

Paulo Ruiz-Grosso ${ }^{1, a}, J$ Jorge Osada ${ }^{1, a}$, Wolfgang Hoppe $^{1, b}$, Bruno Pedraz ${ }^{1, b}$, Johann Vega-Dienstmaier ${ }^{1,2, c}$

Sr. Editor. Según la Norma Técnica de Salud 021-2004 del Ministerio de Salud, el médico de un puesto de salud debe tratar "trastornos depresivos que no requieran manejo especializado". Sin embargo, al analizar los últimos reportes disponibles en las páginas web de las Direcciones de Salud II (Lima Sur) y V (Lima Ciudad) nos percatamos de que los trastornos depresivos no aparecen entre las causas más frecuentes de morbilidad ${ }^{(1,2)}$.

Evidencia científica actual sostiene que la prevalencia de estos trastornos es importante en la población peruana $(6,6 \%)$, más aun al considerar su implicancia en la discapacidad individual ${ }^{(3)}$. Se ha estimado 231820 años de vida saludables perdidos en la población

Grupo de Trabajo en Salud Mental, Universidad Peruana Cayetano Heredia. Lima, Perú.

Hospital Nacional Cayetano Heredia. Lima, Perú

Médico Epidemiólogo; ${ }^{\mathrm{b}}$ Estudiante de Medicina; ${ }^{\mathrm{c}}$ Médico-Psiquiatra

Recibido: 18-08-10 Aprobado: 24-08-11 peruana durante el año $2004{ }^{(4)}$. El hecho de que esto no se refleje en las estadísticas mencionadas, sugiere una baja detección y subregistro en el diagnóstico.

En países desarrollados hasta el $69 \%$ de los pacientes con depresión no son reconocidos por sus médicos tratantes en centros de atención primaria ${ }^{(5)}$, lo cual podría estar replicándose en nuestro medio. El probable subregistro o falta de diagnóstico en atención primaria es importante, pues un diagnóstico y tratamiento oportuno mejorarían el pronóstico en los desórdenes depresivos.

La creciente atención hacia las consecuencias de los desórdenes mentales, especialmente los trastornos depresivos, parece haber tenido poco efecto en la sensibilización de los médicos y demás personal de atención primaria hacia una mejor atención. El manejo oportuno de estos trastornos en el nivel primario de atención, brindaría un beneficio costo-efectividad, más aun cuando se ha descrito que la intervención de un psiquiatra no resulta más eficaz que el manejo dado por un médico general (6). Concluímos que es de interés en salud pública la mejora de la detección, registro y tratamiento de desórdenes depresivos en los primeros niveles de atención.

\section{Conflictos de interés}

Los autores declaran no tener conflictos de interés en la publicación de la presente carta.

\section{REFERENCIAS BIBLIOGRÁFICAS}

1. Ministerio de Salud del Perú, Dirección de Salud Lima II. Morbilidad General por Grupos de Enfermedad. Lima: MINSA; 2011.

2. Ministerio de Salud del Perú, Dirección de Salud Lima V. Primeras 20 Causas de Mortalidad General. Lima: MINSA; 2011

3. Saavedra J. Estudio Epidemiologico en Salud Mental 2002: Informe General. Lima: Instituto Nacional Honorio Delgado - Hideyo Noguchi; 2002.

4. Mitchell AJ, Vaze A, Rao S. Clinical diagnosis of depression in primary care: a meta-analysis. Lancet. 2009;374(9690):609-19.

5. Barbui $\mathbf{C}$, Tansella $\mathbf{M}$. Identification and management of depression in primary care settings. A meta-review of evidence. Epidemiol Psichiatr Soc. 2006;15(4):276-83.

6. Cape J, Whittington C, Bower P. What is the role of consultation-liaison psychiatry in the management of depression in primary care? A systematic review and meta-analysis. Gen Hosp Psychiatry. 2010;32(3):246-54.

Correspondencia: Paulo Jorge Martin Ruiz-Grosso

Dirección: Ca. Ortiz de Zevallos 124, Urbanización San Antonio. Miraflores, Lima 18, Perú.

Teléfono: (511) 2427516

Correo electrónico: paulo.ruiz@upch.pe 